BULLETIN Bulletin hispanique

HISPANIQUE Université Michel de Montaigne Bordeaux

112-1| 2010

Actes du Colloque « langue, littérature, littéralité »

\title{
La Flor de tu secreto
}

\section{Anne-Marie Capdeboscq}

\section{OpenEdition}

\section{Journals}

Édition électronique

URL : http://journals.openedition.org/bulletinhispanique/1184

DOI : 10.4000/bulletinhispanique.1184

ISSN : 1775-3821

\section{Éditeur}

Presses universitaires de Bordeaux

\section{Édition imprimée}

Date de publication : 1 juin 2010

Pagination : 413-432

ISBN : 978-2-86781-692-5

ISSN : 0007-4640

Référence électronique

Anne-Marie Capdeboscq, « La Flor de tu secreto », Bulletin hispanique [En ligne], 112-1 | 2010, mis en ligne le 01 juin 2013, consulté le 06 mai 2019. URL : http://journals.openedition.org/ bulletinhispanique/1184; DOI : 10.4000/bulletinhispanique.1184 


\title{
La Flor de tu secreto
}

\author{
Anne-Marie CapdeboscQ \\ Université de Limoges
}

Comparaisons et métaphores florales construisent sémantiquement et syntaxiquement le discours de la beauté. De Berceo, où le paysage semble se résoudre en équivalences d'ordre spirituel, à Lope de Vega où l'excès du cliché ainsi que sa manipulation syntaxique conduisent à une exacerbation sensorielle et sensuelle, il s'agira, à travers quelques exemples, d'analyser les métamorphoses des métaphores florales.

Comparaciones y metáforas florales construyen semántica y sintácticamente el discurso de la belleza. De Berceo, en cuyos poemas los paisajes se transforman en equivalencias de orden espiritual, a Lope de Vega en que la exaltación de los clichés y su manipulación sintáctica desembocan en la exacerbación sensorial y sensual, se tratará de analizar las metamorfosis de las metáforas florales.

Flower-related comparisons and metaphors both semantically and syntactically build the discourse on Beauty. From Berceo, in whose works scenery seems to be resolved into spiritual equivalences, to Lope de Vega whose extensive use of clichés - as well as their syntactical manipulations - lead to a sensory and sensual exacerbation, the issue of the present article will be analyzing the metamorphoses of floral metaphors throughout a few examples.

Mots-clés: Poésie - Métaphore - Cliché - Mythe - Amarilis.

$B H i$, Tome 112, nº 1 - juin 2010 - p. 413 à 432. 


\section{PrÉSEntation : LE SECRET DU TITRE DONNÉ À LA COMMUNICATION}

$\mathrm{I}$

L PEUT sembler étrange d'emprunter à Pedro Almodóvar, avec une légère modification, le titre d'un de ses films.

La lecture des travaux de Nadine Ly ne laisse pas apparaître, à ma connaissance, des recherches en littéralité filmique. Et pourtant... En laissant mon imagination jouer entre le texte filmique, les textes de Nadine Ly et ceux de Garcilaso, Fray Luis de León, Berceo ou Lope de Vega, j’ai senti se construire des constantes, le réseau du sourire et celui du plaisir de lire et d'écrire. Sourire de la béatitude de Berceo devant la beauté déployée de l'univers créé ; sourire et plaisir de transmuer la tristesse en poésie, de créer la beauté en métamorphosant par les mots l'émotion, en la contraignant à se former en octavillas, en sonnets, églogues, élégies, etc. Il y a un lien certain entre l'harmonie de l'univers créé que chante la cuaderna vía: beauté que disent les fleurs et les pierres précieuses, et le dépassement du désordre introduit par la mort ou la discorde ("Rosa blanca " de Lope de Vega) mais transcendé par l'élaboration qui sublime mort ou désordre (au sens alchimique du terme) ou bien en extrait le sens.

C'est ainsi, qu'en partant de l'œuvre, à mon sens, la plus achevée et la plus ouverte d'Almodóvar : La flor de mi secreto, j'ai été amenée ou ramenée à la réflexion engagée par Nadine Ly autour de La Circe, à ce réseau de connivences qu'établissent les nombres et les noms, à l'infinie transmutation de toute démarche artistique en une autre, au chiffrage des secrets. Il s'agira, dans cet hommage à la générosité intellectuelle de Nadine Ly, d'un prolongement, trop léger sans doute, à ses travaux sur Amarilis.

\section{La flor de mi secreto (1995)}

Dans ce film, comme on le sait, le personnage central féminin, Leo, dont le prénom peut se lire comme l'abréviation de Leocadia mais aussi comme la première personne du singulier du verbe leer, est dédoublé en Amanda Gris, auteure de romans sentimentaux à succès pour le compte de la société Fascination. L'emblème qui figure sur l'Anthologie de ses œuvres est une rose de couleur rose naturellement. En tant que Leo, elle juge sévèrement son double, Amanda Gris, dont la mièvrerie l'exaspère, alors qu'elle-même vit une situation digne d'Amanda Gris (jalousie, trahison, rupture). Almodóvar a créé, avec ce film, une sorte de synthèse de toutes les manifestations par lesquelles l'émotion se dit. L'aspect mélodramatique 
est si soigneusement élaboré qu'une distance souriante ne peut manquer de s'instaurer entre le dire et le dit. Tout dans l'organisation des signaux (décors, codes chromatiques, musique) est icône. Il y a, dans les décors, une insistance sur les fleurs : des tableaux les représentent; on trouve des bouquets savamment disposés : roses de couleur rose, roses rouges, marguerites, jonquilles, rose rouge solitaire dans un vase qui signifient, dans leur disposition et leur succession, peut-être aussi dans le langage naïf des fleurs, les états émotionnels de l'héroïne : de l'attente plus ou moins tranquille au désespoir, de la passion à la tendresse apaisée. Les vêtements sont également en concordance avec l'émotion : beige, bleu, rouge, vert. L'apaisement final qui s'instaure entre le héros masculin, le bien nommé Ángel, et Leo-Amanda est scellé par les deux couleurs complémentaires de leurs vêtements : le rouge et le vert (dont Lope de Vega célèbre, on le verra, la beauté). D'autres élaborations de l'émotion ponctuent le texte filmique : la douleur d'être rejetée est signifiée dans quatre élaborations sémiotiques successives : chaos de la tentative de suicide de Leo d'abord, puis concours de cris retransmis à la télévision; chanson désespérée de Chavela Vargas et enfin manifestation bruyante et rythmique (organisée par conséquent) des étudiants en médecine. Ces élaborations sont autant de mises en forme, de textualisations du beau, de la douleur, de l'amour. L'activité des dentellières à Almagro est également bien sûr la mise en forme de fils, c'est-à-dire encore la textualisation du beau. On trouve également la poésie, récitée par la mère ; la danse gitane qui informe la passion. Tout ceci dans l'écriture filmique, en hommage à tous les langages.

Je terminerai ce survol de La flor de mi secreto par le choix des prénoms.

Leocadia d'abord, Leo = 'je lis', le 'lion' ; Leocadia est le prénom de la femme avec laquelle Goya passa les dernières années de sa vie à Bordeaux et dont il fit, en 1821, un portrait assez noir. C'est aussi le prénom de l'héroïne de La fuerza de la sangre de Cervantès, et j'en oublie certainement. Amanda, le pseudonyme de Leo : celle qui doit être aimée, contient 'amar', comme nous le trouvons dans le prénom Amarilis; Gris, le nom "propre ", se passe de commentaire tant il est le mélange du noir et du blanc. On apprend que la mère de Leocadia porte un nom floral et mythologique : Jacinta (forme féminisée du jacinto de la famille des liliacées). Et pour finir, la cuisinière gitane de Leo prénommée Blanca, dont les vêtements déclinent l'absolu du rouge dans l'apothéose de la danse du spectacle flamenco. Dans ce film total tout signifie et montre la convertibilité infinie des langages : récits, objets, couleurs, fleurs, activités artistiques humaines, pour dire l'émotion esthétique ou autre. 


\section{FLORILÈGE}

Le narcisse, la jacinthe, le crocus, l'anémone, l'héliotrope, la rose rouge, eurent des origines prodigieuses dans un univers où tout avait un sens et une place, une origine, dans un mouvement de compensation permanente où, quand on perd, par exemple, sa qualité de nymphe ou de simple mortel, on peut transcender les limites entre genres et règnes en devenant plante, fleur, fleuve... Cette métamorphose peut être compensatrice, punitive ou protectrice. Cela dépend du dieu ou de la déesse que l'on a offensés ou bien qui pleurent la mort de l'être cher. Le bel Adonis, extrait de l'arbuste Myrte, et fruit des amours incestueuses de Myrrha et de son père Cinyras, fut tué ultérieurement par un sanglier, au grand désespoir de Vénus, et de son sang naquit l'anémone pour certains, dont Ovide, la rose rouge selon d'autres. Myrrha avait été condamnée à mort par son père, coupable d'inceste malgré lui, mais elle fut transformée en arbuste et de ses larmes coule la myrrhe, selon Ovide ${ }^{1}$. La rose rouge porte ainsi le double signe du désir et de la violence. Hyacinthos, aimé par Apollon et Zéphyr, fut tué par erreur par Apollon. De son sang jaillit la fleur qui porte son nom, une sorte d'iris peut-être, dorénavant jacinthe. On connaît bien sûr le destin de Narcisse, puni pour son rejet de la nymphe Echo, et qui devint la fleur qui porte son nom.

Le Cantique des Cantiques ne retient que peu de fleurs alors qu'il insiste sur les arômes et les encens : lis, narcisse, rose peut-être (La rose de Saron également traduite par narcisse de Saron (crocus de Judée). Les textes poposent essentiellement le lis (ou lys), dans lequel les troupeaux de l'aimé paissent et qui s'oppose au chardon ou aux épines. Ce lis, on le retrouve en espagnol sous le double nom d'azucena et de lirio (lilium). On peut ainsi le trouver en compagnie de la rose dans la traduction proposée par Fray Luis de León ${ }^{2}$, El Cantar de los Cantares en octava rima:

\footnotetext{
Esposa: Yo soy rosa del campo muy hermosa y azucena del valle muy preciada.
}

1. Ovide, Les Métamorphoses, Livre X, Paris, GF-Flammarion, 1966, p. 261-274. Pierre Grimal, dans son Dictionnaire de la mythologie grecque et romaine, PUF, 1958, p. 13, rappelle les légendes florales attachées à l'histoire d'Adonis : «non seulement l'origine mythique de la myrrhe (les larmes de Myrrha), mais celle de la rose : à l'origine la rose était blanche, mais comme Aphrodite courait au secours de son ami blessé, elle se piqua le pied à une épine, et la couleur de son sang colora les fleurs qui lui sont consacrées. Les anémones, aussi, passent pour être nées du sang d'Adonis blessé ".

2. Fray Luis de León, Poesías, Barcelona, Planeta, 1970. 
Esposo : Cual entre las espinas es la rosa, tal entre las doncellas es mi amada.

\section{Berceo : Milagros de Nuestra Señora}

On rencontre les fleurs, indifférenciées et odorantes chez Berceo ${ }^{3}$, en tant que catégorie générique composant un paysage idéal qui s'effacera au nom de l'allégorie. Le locus amonus, tempéré en hiver et frais en été, qui intègre dans sa composition l'eau, les arbres, devient un lieu merveilleux, une corne d'abondance inépuisable et infinie, signe d'une fertilité multipliée : pour une fleur que l'on cueille il en repousse trois ou quatre. Dans les strophes d'introduction, Berceo décrit ce lieu merveilleux en insistant sur le parfum des fleurs :

La verdura del prado, la olor de las flores, las sombras de los árbores, de temprados savores, resfrescáronme todo e perdi los sudores:

podrié vevir el omne con aquellos olores [...]

Los omnes e las aves, quantos acaecién, levavan de los flores quantas levar querién, mas mengua en el prado ninguna non façién: por una que levavan tres e quatro nacién.

Les fleurs signifient la vitalité, la croissance infinie, l'immortalité et l'éternité, hors de la temporalité propre aux humains...

Le poète révèle ensuite ce qu'est ce jardin merveilleux ; il ôte l' " écorce » des mots :

\section{tolgamos la corteza, al meollo entremos,} prendamos lo de dentro, lo de fuera dessemos [...]

puis il pose un réseau d'équivalences : ces fleurs sont les noms que les écrits donnent à sainte Marie :

3. Gonzalo de Berceo, Milagros de Nuestra Senöra, Madrid, Cátedra, col. "Letras hispánicas », 1988. 
Le pré, les fleurs, en un mot le lieu, signifient la présence du divin.

$\mathrm{Au}$ désignateur rigide et unique que constitue le prénom Marie se substituent d'autres noms que le poète énumère à la suite : syntagmes nominaux, substantifs. Si le poète nous demande de " laisser " les éléments constitutifs du paysage construit au bénéfice d'autres désignations de ce prénom pourtant désigné, faut-il pour autant renoncer aux fleurs, aux arbres, aux sources, aux oiseaux qui constituent ce "prado " merveilleux ? Certes, on retrouve, en suivant les recommandations de Berceo, la démarche heuristique qui fait aller du connu et objectivement partagé vers le plus abstrait et le moins partagé. Si l'on s'arrête sur l'équivalence signifiée par le verbe copule ser qu'emploie Berceo, on peut faire deux observations complémentaires. En premier lieu, le transfert opéré par ser permet d'ajouter les qualités spécifiques des fleurs à celles des noms de Marie, puis d'ôter l'échafaudage et ne conserver que l'œuvre achevée : les noms. Le créneau syntaxique dans lequel s'insère la métaphore est le plus simple qui soit : de nom à nom. Selon ce principe, l'élaboration allégorique fait des fleurs le véhicule par lequel on s'ouvre à la pluralité des noms de Marie. Les fleurs odorantes seraient donc allégoriquement des noms que l'on révèle, elles constitueraient le langage crypté de la vérité. On peut rejoindre sur ce point les analyses de Paul Zumthor ${ }^{4}$ sur l'allégorie :

Initialement, l'allégorie est mode de lecture. [...] Si le sens est dans les choses, la vérité, elle, n’y réside pas. La vérité reste paradigmatique; le sens se déroule syntagmatiquement. Le langage communique le second, mais voile la première. Il exige donc une double intellection, afin de manifester le lien qui les unit. L'intelligence des mots qui disent les choses permet de saisir leur "sens littéral"; par la voie de l'analogie, l'intelligence du sens littéral fait accéder au sens " allégorique ».

Le lien qui unit fleurs et noms de Marie, du fait de l'emploi premier « des mots qui disent les choses ", permet de saisir leur sens littéral et d'accéder ensuite au sens conçu comme vérité. Mais cette vérité n'est pas de l'ordre du concept, de l'intellect, au contraire, elle relève du visible, du sensible, de tout ce qui, ici-bas, est témoignage, signature et surtout présence de l'au-delà. Ces autres noms de Marie, multiples, infinis, Berceo en énumère quelques-

4. Paul Zumthor, Le masque et la lumière, Paris, Éditions du Seuil, coll. « Poétique », 1978, p. 79. 
uns qui nous engagent dans la nouvelle quête d'un butoir impossible sur lequel s'achèveraient la ronde et la prolifération des substituts. Je n'en citerai que quelques-uns : " la benedicta Virgen es estrella clamada; Ella es vellocino que fue de Gedeón; es uva, almendra, malgranada, oliva, cedro, bálssamo, palma, piértega, fust, bastón. " Et on ne peut que conclure, avec Berceo, qu'il s'agit d'un puits sans fond :

Sennores e amigos, en vano contendemos, entramos en grand pozo, fondo no'l trovaremos; más serién los sus nomnes que nos d'ella leemos que las flores del campo, del más grand que savemos.

La puissance du langage s'impose sur les objets créés du monde naturel.

Ceci dans une première approche car si on analyse la métaphore comme un transfert, la question demeure de l'orientation de ce transfert et en particulier avec l'emploi du verbe copule ser. Ici flores $\longrightarrow$ nomnes de Santa María. Il ne s'agit pas de : nomnes de Santa María = flores. Si l'on y regarde de plus près, on se rend compte que le terme qui est posé en premier, à définir, c'est flores et non pas nomnes. Le 'est' d'équivalence ou verbe copule semble, mais semble seulement, aller du référent du monde naturel vers un signifiant inconnu, aux contours indéfinissables. La nomination des 'noms' passant alors par la nomination de 'choses' du monde naturel. Cette démarche allégorique est en réalité à double orientation : elle élucide (stricto sensu) une notion, une conceptualisation et elle révèle de la réalité physique la conceptualisation qui en fut à l'origine. Élucidation et épiphanie sont les deux faces du double processus d'intellection et de manifestation. Ces nomnes flores se déclinent ensuite à nouveau en estrella clamada, etc. La chaîne synonymique se corrige d'elle-même car, comme dit par Berceo, si l'on saisit une fleur, trois ou quatre renaissent. Si l'on saisit un nom, trois ou quatre surgissent : insaisissabilité... ; absence de limites, mais aussi unité profonde dans la diversité du monde créé.

En effet, la métaphore se résout en métamorphose. Il y a un va-et-vient, herméneutique et pragmatique à la fois, entre l'objet et le nom. Aux fleurs innombrables, saisies globalement, correspond l'infinité des noms de Marie. Aucun hyponyme ne vient restreindre le domaine de projection car, par rapport à la métaphore élémentaire qui court de Nom à Nom avec la médiation d'un déterminant particularisant, selon le modèle : "Richard est un lion", la métaphore ne se construit pas en restreignant le comparant par le déterminant " un " et le comparé à un seul individu, mais, au contraire en passant par les déterminants universalisants. Las flores son los nomnes est 
réversible en «los nomnes son las flores ». Et Marie a de multiples avatars, dont les fleurs et l'infinité des noms.

\title{
Fray Luis de León ${ }^{5}$
}

\author{
Canción de la vida solitaria \\ Del monte en la ladera, \\ por mi mano plantado, tengo un huerto, \\ que con la primavera, \\ de bella flor cubierto, \\ ya muestra en esperanza el fruto cierto; \\ (v. 41-45)
}

Ici, le paysage rêvé est construit par une saisie générique de la bella flor des arbres fruitiers. Le huerto florifège est essentiellement fructifere car la promesse des fruits est promesse de vie éternelle. Le poète associe ensuite la fontana pura à la beauté (hermosura) du huerto qui s'anime de fleurs :

$$
\begin{aligned}
& \text { y, como codiciosa } \\
& \text { por ver y acrecentar su hermosura, } \\
& \text { desde la cumbre airosa } \\
& \text { una fontana pura } \\
& \text { hasta llegar corriendo se apresura; } \\
& \text { y, luego sosegada, } \\
& \text { el paso entre los árboles torciendo, } \\
& \text { el suelo, de pasada, } \\
& \text { de verdura vistiendo } \\
& \text { y con diversas flores va esparciendo. } \\
& \text { (v. 51-55) }
\end{aligned}
$$

Noche serena

$$
\begin{aligned}
& \text { ¡Ay, levantad los ojos } \\
& \text { aquesta celestial eterna esfera! }
\end{aligned}
$$

L'injonction des vers 31 et 32 incite à dépasser la condition terrestre, la fragilité des hommes. À partir du vers 66, se construit le paysage fleuri d'un

5. Fray Luis de León, Poesias, Madrid, Cátedra, col. « Letras hispánicas », 1997. 
printemps qui syncrétise l'éternité, c'est-à-dire la conjonction des contraires par l'annulation des contraintes propres aux référents, ici contrôlés par les adjectifs qui en font éclater les limites :

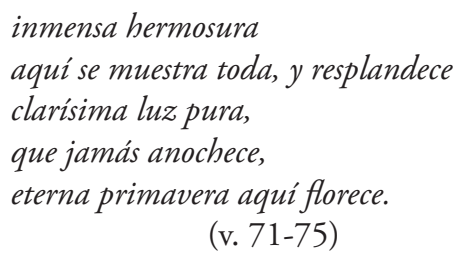

La restriction temporelle liée au printemps est abolie par l'adjectif eterna, de même que la luz est toujours claire. Tant jamás que eterno sollicitent leur contraire : "toujours "; " éphémère ". La nuit succède toujours au jour ; le printemps n'a qu'un temps, qu'une saison. Dans la stylisation du paysage extraordinaire se dit la beauté du monde céleste, qui sera transportée en " vérité » dans la strophe suivante :

$$
\begin{aligned}
& \text { ¡Oh campos verdaderos! } \\
& \text { ¡oh prados con verdad frescos y amenos! }
\end{aligned}
$$

Par ce mot abstrait qui reformule la inmensa hermosura du premier vers de la strophe précédente nous passons de l'esthétique, de la plénitude sensorielle à la modalité épistémique de la vérité. La beauté est vérité. Elle est 'immense' car elle est sans limites et ne peut être mesurée. On a souvent souligné que les réalités du monde créé, telles que primavera, sont immédiatement déréalisées, c'est-à-dire extraites de leur contingence temporelle grâce au syntagme qui les accueille. Ainsi, dans De la vida del Cielo, l'éphémère de la rose est-il immédiatement annulé par le prédicat inmortal:

$$
\begin{aligned}
& \text { él va y en pos dichosas } \\
& \text { le siguen sus ovejas, do las pace } \\
& \text { con inmortales rosas, } \\
& \text { con flor que siempre nace } \\
& \text { y cuanto más se goza más renace. }
\end{aligned}
$$

Il n'y a ni couleur ni odeur dans ces représentations. À une exception près. Le titre du poème de Fray Luis Las serenas [ou sirenas] est d'ailleurs un subtil avertissement : 


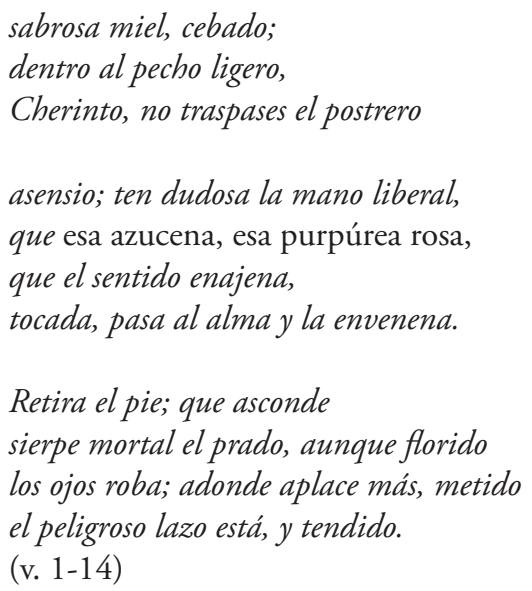

Les deux fleurs sélectionnées pour leur beauté et leur séduction, azucena et purpúrea rosa, empoisonnent l'âme car elles peuvent captiver les sens. Le poète regarde vers le bas, vers la terre qu'il ne faudrait pas fouler. C'est le plaisir qui est dangereux, l'enivrement sensoriel. La séduction fatale de ces fleurs est symbolisée plus bas dans le poème par Circé, la sorcière :

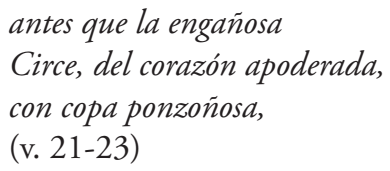

La chaîne analogique se construit ainsi : purpúrea rosa $\longrightarrow$ veneno $\longrightarrow$ prado mortal $=$ lazo peligroso $=$ Circe.

$\mathrm{Au}$ terme de ce très rapide parcours, on s'aperçoit que la fleur est essentiellement convoquée pour ce qu'elle n'est pas, c'est-à-dire fugace : tantôt symbolisation de l'éternité et de la Béatitude, tantôt concentré de la périlleuse beauté du monde.

\section{Avènement de la rose et d'autres fleurs}

La rose devient la fleur par excellence, l'hyperonyme de fleur. Elle est, chez Santillana ${ }^{6}$, mise en relation avec la jeunesse, la beauté et l'amour. Elle

6. Marqués de Santillana, Poesías completas, I, Madrid, Castalia, 1975. 


\section{LA FLOR DE TU SECRETO}

sert de parure végétale aux bergères rencontrées et devient l'aune à laquelle elles se mesurent avec succès.

Serranilla VI:

$$
\begin{aligned}
& \text { En un verde prado } \\
& \text { de rosas y flores, } \\
& \text { guardando ganado } \\
& \text { con otros pastores, } \\
& \text { la vi tan graciosa, } \\
& \text { que apenas creyera } \\
& \text { que fuese vaquera } \\
& \text { de la Finojosa. }
\end{aligned}
$$

Non creo las rosas de la primavera sean tan fermosas nin de tal manera; fablando sin glosa, si antes supiera de aquella vaquera de la Finojosa.

Serranilla IX
Mas vi la fermosa de buen continente, la cara plaçiente, fresca como rosa, de tales colores qual nunca vi dama nin otra, señores.

De même dans le Villancico suivant :
Por una gentil floresta de lindas flores [e] rosas, vide tres damas fermosas que de amores han requesta. $[\ldots]$

Dans ces rosas y flores, la rose semble à part, elle est exceptionnelle, absolue car nommée et son nom est le seul à rimer inévitablement avec fermosa(s). Elle est présentée comme l'hyperonyme qui ferait des fleurs de 
simples hyponymes. La rose eut longtemps le privilège d'orner, seule, les prés ombreux et herbeux. Beauté, perfection. Dès Santillana, le rapport s'inverse : la rose sert de comparant de la femme qui la surpasse en beauté. Puis elle sera concurrencée par d'autres fleurs. La femme se métamorphose en fleur. Et la fleur contribue à construire en poésie le corps féminin et le plus souvent le visage féminin.

Le sonnet XXIII de Garcilaso témoigne de la modification intervenue, en une sorte de retour à la réalité. Il y a une destinataire, la femme, dans ce Carpe Diem réécrit : mais la color de vuestro gesto es de rosa y d'azucena. De même que la chevelure est d'or : le poète emprunte à la nature des éléments par une démarche non d'équivalence mais d'extraction de propriétés. Les propriétés de la beauté impliquent sa dégradation : beauté et destruction ; beauté et laideur qui sont la définition même de l'éphémère de la vie et de la temporalité.

On sait le sort que Góngora ${ }^{7}$ réservera à ce motif. J'en soulignerai seulement l'enrichissement botanique par rapport à la tradition horatienne suivie par Garcilaso :

Mientras por competir con tu cabello, oro brunido el Sol relumbra en vano, mientras con menosprecio en medio el llano mira tu blanca frente al lilio bello;

mientras a cada labio, por cogello, siguen más ojos que al clavel temprano, y mientras triunfa con desdén lozano de el luciente cristal tu gentil cuello; goza cuello, cabello, labio y frente, antes que lo que fue en tu edad dorada oro, lilio, clavel, cristal luciente

no sólo en plata $o$ víola troncada se vuelva, mas tú y ello juntamente en tierra, en humo, en polvo, en sombra, en nada.

Lilio bello, clavel temprano, seront conjoints par les ravages du temps en viola troncada, puis absorbés par le terrible indéterminé ello du vers 13 qui précède le néant final.

7. Luis de Góngora, Obras completas, Madrid, Aguilar, 1956, Soneto 228. 
Pour conclure ce bref tour d'horizon de ce que la poésie doit à la diversité des fleurs, je rappellerai que le prénom Amarilis, dont il va être question, n'est pas la seule propriété de Lope de Vega. Quevedo, par exemple, dans ses Poemas amorosos, évoque Amarilis mais aussi Floris, Flora, Florisa, Flori, Rosalba, Jacinta et bien sûr Lisi, la féminité étant toujours associée aux fleurs.

\section{LOPE DE VEGA}

L'enrichissement botanique du répertoire amoureux est manifeste chez Lope de Vega et il va de pair avec une observation minutieuse des fleurs qui légitime, en quelque sorte, les analogies. Si auparavant, la fleur et par excellence la rose étaient invoquées comme absolu de beauté ou de fragilité, maintenant le poète s'applique à mettre en rapport les éléments de la fleur (le comparant) avec les parties du visage ou les modalités expressives à exalter. Ainsi dans Égloga a Amarilis ${ }^{8}$ le berger Elisio construit-il un portrait d'Amarilis où l'éclosion de la mauve entre en consonance avec les joues de la bergère, ajoutant le mouvement à la couleur :
Como se mira doble malva abriendo
del cerco de hojas en carmin fogoso,
asi de las mejillas sobre nieve
el divino pintor púpura llueve.

Pour évoquer l'arrivée d'Amarilis dans le tournoi qu'elle doit présider, c'est la rose qui est sollicitée avec ses sépales verts auxquels se substituent "verdes rayos":
Como sale después de noche escura
la pura rosa en el botón compuesta
de aquel pomposo purpurante adorno
de verdes rayos coronada en torno.

Dans le Livre I de La Arcadia9 (1598), Alastio rend hommage à la beauté de Crisalda en confrontant systématiquement un impressionnant registre floral à sa beauté et aux éléments canoniques de son corps (frente, mejillas, labios, cuello, manos, cabellera, venas, pechos et même aliento) :

8. Lope de Vega, "Égloga a Amarilis", in Obras no dramáticas de Fray Lope Felix de Vega Carpio, Madrid, B. A. E., tomo XXXVIII, 1950.

9. Lope de Vega, "La Rosa blanca », Obras completas, », Barcelona, Planeta, 1983. 


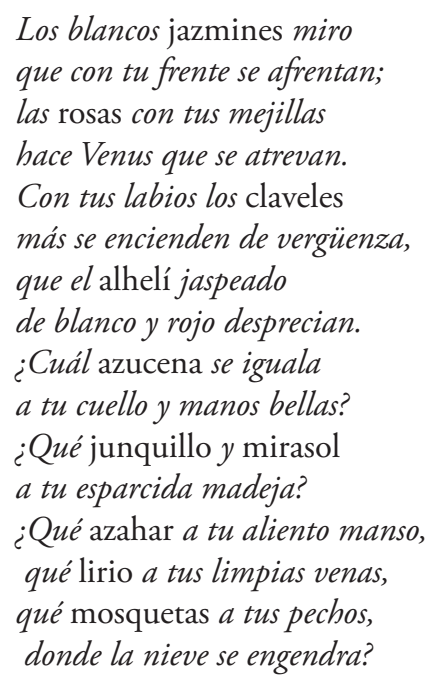

Le poète condense lui-même ce répertoire pour démontrer que la beauté humaine défie la beauté florale :

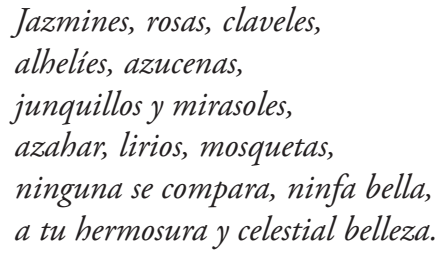

Le paradoxe de la négation, du déni de toute comparaison possible entre fleurs et beauté féminine, fait que s'actualise dans cette composition un étrange portrait, figurativement proche des compositions d'Arcimboldo.

C'est le saisissant passage brutal et rapide de la beauté à la mort qui sera emblématisé dans la création mythique de la rose rouge, comme résultat d'une conversion ou métamorphose absolue.

\section{Les métamorphoses de la rose}

Dans Littéralité 2, "La Circe de Lope de Vega ", Nadine Ly ${ }^{10}$ consacre des pages remarquables à La Rosa Blanca, poème mythologique de Lope de Vega qui narre la naissance, puis les métamorphoses de la rose. Je la cite :

10. Nadine Ly, Littéralité 2, "La Circe de Lope de Vega ", Bordeaux, PUB, 1992, p. 151180. 


\section{LA FLOR DE TU SECRETO}

La rose blanche, doublement emblématique, pour être rose et pour être blanche, par son aptitude à signifier le centre de la Croix, le cour du Christ, la rose mystique enfin, en relation avec la déesse de l'amour universel et de l'harmonie du monde, Vénus, et avec l'archaïque Circé, est le véhicule de ce passage de l'amour charnel évité à l'amour sacré.

Dans cette communication, Nadine Ly démontre que le nom d'écriture de Marta de Nevares, Marcia Leonarda, Amarilis, est le qualifiant exclusif de la rose blanche. Je vais emprunter à mon tour le chemin mythologique et poétique des incarnations successives de la fleur en suivant le poème de Lope de Vega.

D'abord, la rose fut rouge ${ }^{11}$, née du sang de Vénus qui s'était blessé le pied sur des épines (zarzas) : "Nació encarnada del rubi sangrientolque de Venus vertió la planta herida; Ino fue primero blanca... ".

Selon Lope de Vega, qui suit ainsi Ovide, la poursuite amoureuse de Mars fut la cause de cet " accident » :
De aquella sangre procedió la rosa, en verde silla de un botón sentada, con cinco guardas, que su pompa hermosa tienen, cuando se extiende coronada; abrió por muchas hojas olorosa la boca en tierna puirpura bañada, mostrando dentro, para más decoro, en vez de blancas perlas, granos de oro.

Cette rose première était anthropomorphisée et présentée comme une reine sur le trône de son vert bouton (associant ainsi les deux couleurs vert et rouge) ; elle avait 5 gardes (les 5 sépales auparavant appelés rayos dans "Égloga a Amarilis ") et surtout une bouche : la boca en tierna púrpura bañada. À la place des dents, souvent qualifiées poétiquement de perles, on admire des grains d'or : en vez de blancas perlas, granos de oro. Le sang et la douleur de Venus blessée. La cohérence est totale entre la morphologie du référent « rose » et la figure féminine qu'elle suggère. Lope s’appuie sur le topos du portrait féminin dans lequel les fleurs intervenaient soit comme parures

11. On a noté plus haut que pour Ovide la fleur en question était une anémone. Pierre Grimal rappelait de son côté que la rose était initialement rose avant d'être teintée du sang de la déesse Vénus. 
(Garcilaso) soit comme faire-valoir des propriétés esthétiques (comme dans l'exemple cité plus haut de La Arcadia), ce topos étant un modèle rhétorique ouvert à de subtiles variations. Mais il s'applique également à faire coïncider la représentation de l'avènement de la rose avec une morphologie détaillée de la fleur dont chaque élément est présent métaphoriquement, à l'exception des hojas : verde silla = bouton; cinco guardas = sépales; granos de oro = étamines. Il n'est jusqu'à la corolle (étymologiquement 'petite couronne') qui ne soit transcendée par le 'coronada' de la rose épanouie. La rose rouge est chargée des ornements royaux : pompa; coronada ; purpura. C'est dire que nous nous trouvons face à la métaphorisation de la fleur par des éléments humains. On peut ajouter que l'orientation du processus métaphorique est incertaine : la rose royalement belle est-elle métaphorisée par traduction de ses éléments constitutifs (calice, sépales, corolles, étamines...) en qualificatifs royaux ou est-ce la fleur qui se voit ainsi décrite parce qu'ainsi perçue? Sens littéral et sens figuré se confondent. L'observation des sépales des roses qui constituent le calice a-t-elle favorisé d'abord une description en termes de botanique, métaphorisés ensuite, ou bien, le poète n'a-t-il pas immédiatement perçu des gardes (guardas), un trône (silla), une tête couronnée (coronada) ?

Pour reprendre la lecture " alchimique » proposée par Nadine Ly, ici nous sommes face à une "rubification » initiale, que rien, sur le plan alchimique ne justifie. Le rouge qui contient déjà des grains d'or (granos de oro) est la troisième phase du processus, qui vient après l'albedo (l'œuvre au blanc). Et le poème nous fait reculer dans le processus, le reprendre presque à zéro, pour aboutir au 'blanc'.

En effet, la rose rouge est si belle que Vénus et Junon s'affrontent pour la posséder. Vénus surtout qui est une sorte d'incarnation divine de la rose :

$$
\begin{aligned}
& \text { Venus, moviendo el amoroso labio, } \\
& \text { cuyo coral con tantas perfeciones } \\
& \text { a la rosa imitó, que parecía } \\
& \text { que buscaba lo mismo que tenía. }
\end{aligned}
$$

La querelle dégénère et chacune s'arme pour une bataille qui s'annonce sanglante : rupture de la sérénité du ciel, colère et, comme le redoute Jupiter, discorde (1) (séparation) = rupture d'unité ; dissonance. C'est-à-dire le stade 1 de l'opération alchimique : l'œuvre au noir (appelé également putréfaction) : « si tu ne noircis pas, tu ne blanchiras pas ", disait l'alchimiste Nicolas Flamel. Jupiter fait cesser cette discorde en enfermant la rose (rouge) dans un temple consacré à la déesse Flora. Elle y est vénérée (chastement) par les nymphes auxquelles elle offre sa couleur et sa flamme. Parmi les nymphes 
se détache Amarílida (" tomó la rosa que en el templo ardía, con la color que en púrpura bañaba »). Et c'est ainsi que la chaste et " cándida ", blanche nymphe Amarilida divina prie tant la rose qu'elle en acquiert la splendide couleur (carmesí, grana). On remarquera que si la rose première était rouge la nymphe Amarílida, elle, était blanche. Jupiter tombe amoureux d'elle, et quelles que soient les apparences sous lesquelles il sollicite Amarilida,

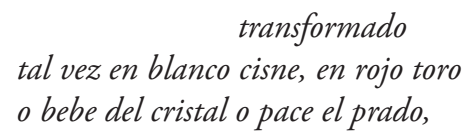

il échoue. La belle résiste car elle est fiancée à un berger. C'est un nouveau désordre (2) qui est ainsi introduit. Junon intervient et transforme la rose rouge, enfermée dans le temple mais qui propageait sa couleur à la nymphe, en rose blanche, ou plus exactement en neige qui, naturellement, éteint tant le feu de la rose rouge que sa couleur. Et, bien sûr, atteint également Amarílida, la nymphe, dont le destin se confond avec celui de la rose.

$$
\begin{aligned}
& \text { y transformóla en nieve blanca y pura } \\
& \text { por quitar el color a la hermosura. }
\end{aligned}
$$

C'est l'albedo enfin. "Opération de la purification de la matière ", encore appelée " cuisson de la matière ». Nadine Ly en rappelle la signification dans notre poème :

L'Albedo, opération de purification de la matière et de spiritualisation, est soutenue par l'institution littéralement inscrite dans le texte, de la procédure rhétorique de métaphorisation ou d'allégorisation, c'està-dire de l'inscription de l' 'esprit' dans la matière, ou du sens figuré dans le sens littéral ${ }^{12}$.

En effet, le passage au blanc (albedo) détermine la résurrection. Manque la troisième phase, la « rubification ", selon Jung ${ }^{13}$ :

À ce stade, le premier résultat essentiel du processus est atteint, on le nomme albedo [...] que beaucoup d'alchimistes estimaient autant que s'il avait été le but dernier du processus. C'est l'état de l'argent ou de la lune, qui doit être encore élevé à l'état du soleil. L'albedo est en quelque sorte l'aube, alors que le rubedo (le rouge) est le lever du soleil.

12. Nadine Ly, art. cit., p. 161.

13. C. G. Jung, Psychologie et Alchimie (1943), Paris, Buchet/Chastel, 1970, p. 303. 
De cette " rubification ", A.-J. Pernety ${ }^{14}$ souligne qu'elle est le terme du processus :

Rubification. Continuation du régime hermétique au moyen duquel on parvient à faire passer la matière de la couleur blanche à la rouge.

Deuxième et troisième phase étaient également appréciées dans leur épiphanie colorée : le rouge et le blanc. Mais la discorde (3) surgit à nouveau car les nymphes se plaignent et Jupiter dote alors la rose de deux couleurs : couleur rouge et couleur blanche. Amarílida bella, naguère telle la rose rouge, devient, comme la rose, bicolore et parvient à l'équilibre de la beauté : la fusion entre la rose et Amarílida est complète :

\section{Amarilida bella, componiendo de rojo y blanco el rostro delicado, las hojas de la rosa repartiendo dejóle en nieve y púrpura bañado}

le tout accompagné de références florales : "jazmin a los claveles añadiendo ». Fleur parmi les fleurs. Le désordre (4), en l'occurrence la jalousie, menace encore car Amarílida (ou la rose) séduit encore et toujours faunes, satyres, silènes... Et Junon accomplit l'acte ultime : elle détruit la rose, en éparpille les couleurs (" lo rojo y blanco van cubriendo el suelo ») signant ainsi le fait que le rouge et le blanc sont mutuellement exclusifs. Séparés et bien distincts. Dorénavant, il y a deux catégories de roses : les roses rouges, nombreuses, et les roses blanches, beaucoup plus rares. Les roses rouges de l'amour et les roses blanches de la pureté. Et c'est ainsi que de la rose initiale, rouge, unique, naquirent les roses.

Lope a prolongé en la dupliquant la querelle Minerve/Vénus autour de la pomme d'or. Les roses sont donc le résultat de quatre transformations : d'abord unique, elle naît du sang de la blessure de Vénus ; la jalousie de Junon en fait une fleur blanche; écoutant les plaintes des nymphes, Jupiter, dans le rôle de conciliateur, la rend bicolore et, enfin, Junon effectue l'ultime opération de séparation, de division en deux catégories de roses. Le destin de la rose c'est le destin d'Amarílida, tant la version de Lope en construit une relation d'osmose et de contamination réciproque.

14. A.-J. Pernety, Dictionnaire mytho-hermétique (1758), Milan, Arché, 1980, p. 443. 


\section{Rose, Amarilis, Amaryllis}

La rose est donc également " un nom d'écriture " (comme on dit un nom de scène) relié à Marcia Leonarda, alias Amarilis, alias Marta de Nevares ${ }^{15}$.

La chevrière de la troisième Idylle de Théocrite, prénommée Amaryllis, a eu une longue carrière poétique, musicale : Théocrite fit naître le personnage d'Amaryllis, du grec " briller ", " resplendir ", chevrière fort belle, indifférente, voire cruelle. On la retrouve dans la première Bucolique de Virgile; Ovide l'évoque brièvement dans L'Art d'aimer. En Espagne, Herrera lui consacre une églogue. C'est Giovanni Battista Guarini qui la relance avec sa tragicomédie Il pastor fido (autour de 1590) en jouant sur l'improbable mais audible étymon : amar du prénom Amarilis. En espagnol, lilio, [lis], vient compléter le programme onomastique car la composition amar-lilio se laisse aisément entendre et l'ensemble Amarilis s'ouvre à toutes les rêveries. Elle habite certains sonnets de Quevedo mais surtout, elle hante la poésie de Lope. Dans La rosa blanca, on vient de voir qu'Amarílida, nymphe dédiée à Diane, est si belle qu'elle déclenche une querelle olympienne et conjugale. Dans la Égloga a Amarilis, le poète en appelle aux roses du matin pour "pintar " sa bouche. La grâce de sa voix "entre clavel " (métonymie de la bouche car l'œillet est toujours poétiquement rouge) et "roja manutisa". Et enfin, lorsqu'elle est amenée à présider une joute poétique, elle apparaît comme (là c'est une comparaison) une rose royale et pure qui stylise et sublime les qualités essentielles visuelles de la rose. Je rappelle les vers qui exaltent sa naissance :
como sale después de noche escura
la pura rosa en el botón compuesta de aquel pomposo purpurante adorno de verdes rayos coronada en torno.

La pourpre de purpurante et l'éclat vert qui l'entoure.

La série est complète : Marta de Nevares = Marcia Leonarda = Amarilis $\longrightarrow$ rose rouge, fragile et mortelle, dont le poète pleure encore la mort dans La Dorotea.

15. Nadine Ly, art. cit., p. 161. 


\section{Amaryllis}

Et la chevrière devint une fleur : dernier avatar.

La rose symbolique ou rêvée des poètes n'était pas un amaryllis.

Amaryllis n'était pas originellement une plante. Juste celle qui brille. Or elle le devint.

C'est Linné qui, en 1753, donna à la plante bulbeuse du genre Hippeastrum le doux nom d'Amaryllis, en honneur à la beauté. Le réseau poétique qui court de Théocrite à Lope de Vega va donner naissance à une plante. L'amaryllis appartient à la famille des Liliacées (comme le Lis, la Jacinthe, le Narcisse). Les botanistes ultérieurs considèrent que Linné avait voulu désigner sous ce nom le Lilium Belladonna - nom qui en soi est également une promesse ! mais le nom est resté car il était beau (ou en hommage à Linné). Peut-être aussi Linné avait-il voulu rétablir une injustice poétique car la jacinthe, le narcisse, étant (dans la mythologie) des mortels devenus végétaux, par la grâce de l'amour, pourquoi la plante que Linné voulait nommer n'aurait-elle pas reçu un nom qui semblait contenir, en latin, 'amare' et 'lilium' ? 